\title{
Heat Kernel Smoothing via Laplace-Beltrami Eigenfunctions and Its Application to Subcortical Structure Modeling
}

\author{
Seung-Goo Kim ${ }^{1}$, Moo K. Chung ${ }^{1,2,3, \star}$ Seongho Seo ${ }^{1}$, \\ Stacey M. Schaefer ${ }^{3}$, Carien M. van Reekum ${ }^{5}$, and Richard J. Davidson ${ }^{3,4}$ \\ 1 Department of Brain and Cognitive Sciences, \\ Seoul National University, Korea \\ 2 Department of Biostatistics and Medical Informatics \\ 3 Waisman Laboratory for Brain Imaging and Behavior \\ 4 Department of Psychology and Psychiatry, \\ University of Wisconsin, Madison, WI, USA \\ 5 Centre for Integrative Neuroscience and Neurodynamics, School of Psychology \\ and Clinical Language Sciences, University of Reading, UK
}

\begin{abstract}
We present a new subcortical structure shape modeling framework using heat kernel smoothing constructed with the LaplaceBeltrami eigenfunctions. The cotan discretization is used to numerically obtain the eigenfunctions of the Laplace-Beltrami operator along the surface of subcortical structures of the brain. The eigenfunctions are then used to construct the heat kernel and used in smoothing out measurements noise along the surface. The proposed framework is applied in investigating the influence of age (38-79 years) and gender on amygdala and hippocampus shape. We detected a significant age effect on hippocampus in accordance with the previous studies. In addition, we also detected a significant gender effect on amygdala. Since we did not find any such differences in the traditional volumetric methods, our results demonstrate the benefit of the current framework over traditional volumetric methods.
\end{abstract}

\section{Introduction}

The amygdala and hippocampus are primary subcortical structures involved in emotion and memory [1]2. Age and gender could be major factors that affect the functions and structures of these regions, as implied by postmortem studies [3]. Although the atrophy of brain tissues associated with the increase of age is reported in several hundreds subjects [45], the findings on the atrophy of amygdalar and hippocampal structures are somewhat inconsistent. The volume reduction of amygdala and hippocampus due to aging has been found in some studies [6/7,8], while other studies did not find such association [4/5,9,10]. For

\footnotetext{
* Corresponding author: Moo K. Chung, Waisman Center \#281, 1500 Highland Ave.
} Madison, WI. 53705, mkchung@wisc.edu, http://www.stat.wisc.edu/ mchung/ 
the effect of gender, one study reported significant differences in amygdala and hippocampus volume between the groups [11] whereas others failed to reproduce these [12]. The inconsistency between these results may have been due to the different image processing and analysis pipelines used in these studies.

In these volumetric studies, the total volume of the amygdala or hippocampus was typically estimated by tracing the region of interest (ROI) manually and counting the number of voxels within the ROI. The limitation of this ROIbased volumetry is that it cannot determine if the volume difference is diffuse over the whole ROI or localized within specific regions of the ROI [13]. Our proposed deformation-based morphometry (DBM) framework can localize the volume difference up to the mesh resolution at each surface mesh vertex.

Using the 3D deformation field derived from spatial normalization of MRI, we can model how the surfaces of subcortical structures are different from each other at the vertex level. Since the deformation field is noisy, it is necessary to smooth out the field along the surface to increase the signal-to-noise ratio (SNR). Further, smoothing is desirable in satisfying the assumptions of the random field theory (RFT), which is used in correcting for multiple comparisons 14 15. For RFT to work, the Gaussianness and smoothness of data are needed [14]16]. As the amount of smoothing increases, Gaussianness and smoothness of data increases. With these motivations, we present a new framework of smoothing scalar and vector measurements using heat kernel smoothing, which is equivalent to performing isotropic diffusion but without discretizing the diffusion equation. The proposed framework is then used in examining the effect of age and gender on amygdala and hippocampus, contrasting the traditional volumetric analysis.

\section{Method}

We analyze the shape of subcortical structures as follows: (1) obtain a population mean volume by averaging the spatially normalized binary masks, and extract a template surface from the averaged binary volume (section 2.1), (2) interpolate the $3 \mathrm{D}$ displacement vector field onto the vertices of the surface meshes (section 2.1), (3) perform heat kernel smoothing on the displacement length along the template surface to reduce noise, and on the surface coordinates to smooth out the surface itself for better visualization (section 2.2 and 2.3), (4) apply a general linear model testing the effect of age and gender. The detailed description of each step is given in section 2 except the statistical inference which is given in section 3 .

\subsection{Images and Preprocessing}

We have high resolution T1-weighted inverse recovery fast gradient echo anatomical 3D images, collected in 124 contiguous $1.2-\mathrm{mm}$ axial slices ( $\mathrm{TE}=1.8 \mathrm{~ms}$; $\mathrm{TR}=8.9 \mathrm{~ms} ;$ flip angle $=10^{\circ} ; \mathrm{FOV}=240 \mathrm{~mm} ; 256 \times 256$ data acquisition matrix) of 69 middle age and elderly adults ranging between 38 to 79 years 

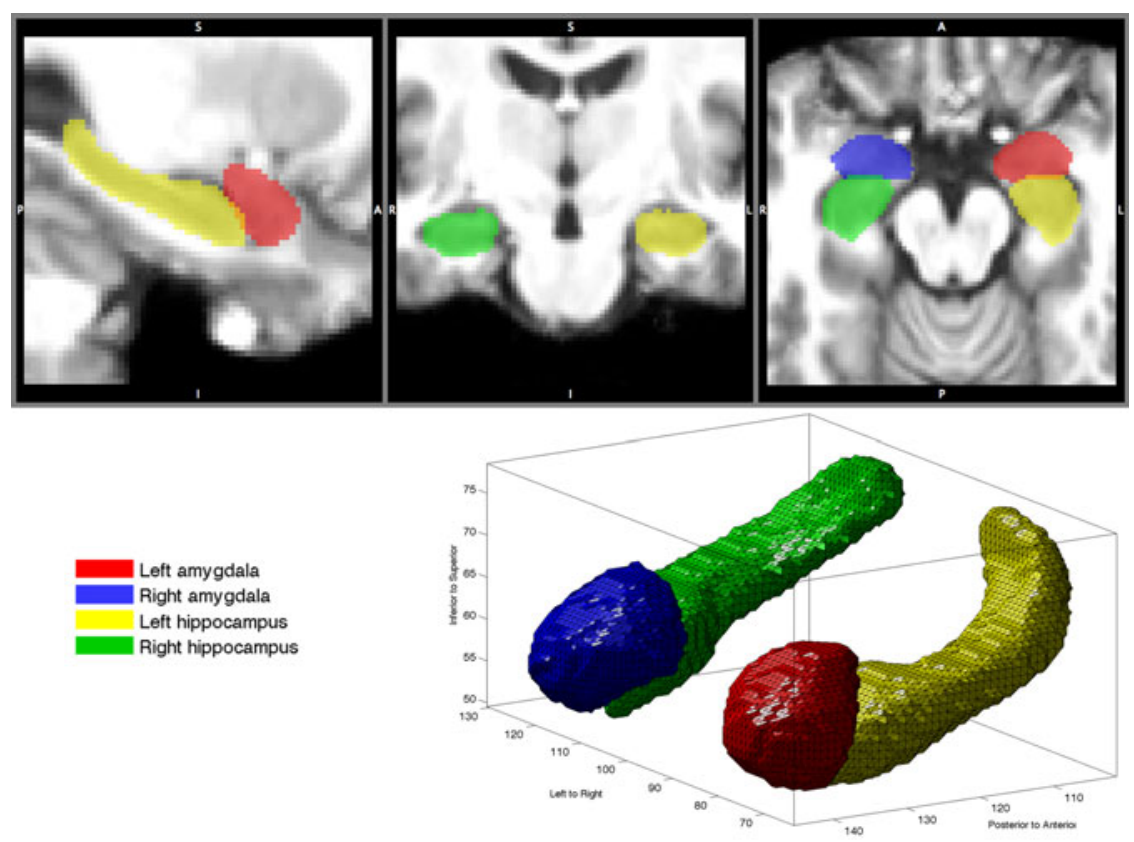

Fig. 1. Subcortical masks superimposed on MRI (top) and the corresponding isosurfaces of the masks (bottom)

(mean age $=58.04 \pm 11.34$ years). The data were originally collected for a national study for the health and well-being in the aged population, called MIDUS (Midlife in US; http://midus.wisc.edu/).

There are 23 males and 46 females. The amygdalae and hippocampi were manually segmented by a trained individual rater. Brain tissues in the MRI scans were first segmented using Brain Extraction Tool (BET) [17. Then we performed a nonlinear image registration using the diffeomorphic shape and intensity averaging technique with cross-correlation as similarity metric through Advanced Normalization Tools (ANTS) [18. A study-specific unbiased template was constructed from a random subsample of 10 subjects. Using the deformation field of warping the individual brain to the template, we deformed the amygdala and hippocampus binary masks to the template space. The normalized masks were then averaged to produce the subcortical masks. The isosurfaces of the subcortical masks are extracted using the marching cube algorithm [19]. The subcortical masks and the corresponding surfaces are shown in Fig. 1.

Using ANTS, we have the deformation vector field of warping an individual brain to the template. The vector field is defined on voxels. On the other hand, the vertices of the subcortical surface meshes are located within voxels. So we simply assigned the vector field onto the mesh vertices by linear interpolation (Fig. 2). The length of the displacement vector at each vertex is computed and used as a feature to measure the local shape variation. 

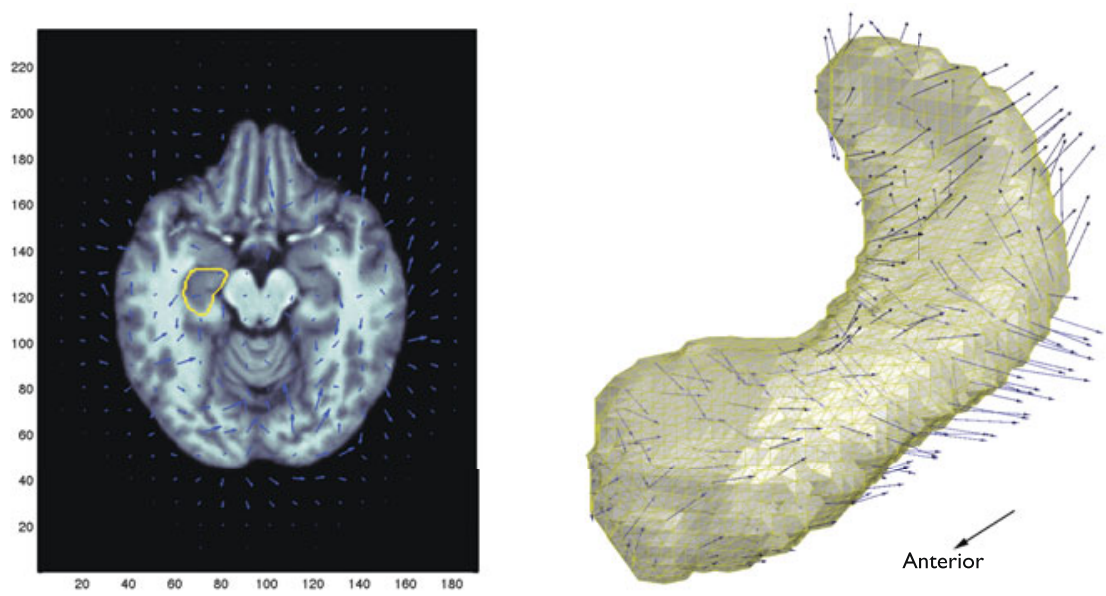

Fig. 2. Displacement vector field (blue arrows) of a subject on an axial slice of the template brain (left). Yellow contour in the left panel is the boundary of the left hippocampus in the template. The vector field has been interpolated on the left hippocampus surface (right).

\subsection{Heat Kernel Smoothing}

Since the displacement length on the template surface is noisy, it is necessary to smooth out the measurements to increase the signal-to-noise ratio (SNR) and to improve the smoothness and Gaussianness of data for RFT-based statistiscal inference [20]. We propose a new diffusion smoothing framework that uses the Laplace-Beltrami eigenfunctions.

Diffusion equations have been widely used in image processing as a form of noise reduction starting with Perona and Malik in 1990 [21. Although numerous techniques have been developed for surface fairing and mesh regularization $\left.\begin{array}{llll|l|l|l|l|l|l|l|l|l}20 & 22 & 23 & 24 & 25 & 26\end{array}\right]$ based on heat diffusion. Most diffusion smoothing approaches mainly use finite element or finite difference schemes which is known to suffer numerical instability if the forward Euler scheme is used.

In this paper, we propose a new smoothing framework that constructs the heat kernel analytically using the eigenfunctions of the Laplace-Beltrami operator. Although solving the eigenfunctions of the Laplace-Beltrami operator requires the finite element method, the proposed method is analytic in a sense that heat kernel smoothing is formulated as a series expansion explicitly. We are not claiming our framework to be analytic which is theoretically impossible when dealing with real data. The proposed method represents isotropic heat diffusion analytically as a series expansion so it avoids the numerical instability associated with solving the diffusion equations numerically [20 22 27]. Our framework is an improvement over previous approaches in the sense that it bypasses the various numerical problems that are associated with previous approaches including numerical instability, slow convergence, and accumulated linearization error. 
Consider a real-valued functional measurement $Y(p)$ defined on a manifold $\mathcal{M} \subset \mathbb{R}^{3}$. We assume the following additive model:

$$
Y(p)=\theta(p)+\epsilon(p),
$$

where $\theta(p)$ is the unknown mean signal to be estimated and $\epsilon(p)$ is a zeromean Gaussian random field. We may assume $Y \in L^{2}(\mathcal{M})$, the space of square integrable functions on $\mathcal{M}$ with the inner product

$$
\langle f, g\rangle=\int_{\mathcal{M}} f(p) g(p) d \mu(p),
$$

where $\mu$ is the Lebesgue measure such that $\mu(\mathcal{M})$ is the volume of $\mathcal{M}$. Solving

$$
\Delta \psi_{j}=\lambda_{j} \psi_{j}
$$

for the Laplace-Beltrami operator $\Delta$ on $\mathcal{M}$, we find the eigenvalues $\lambda_{j}$ and eigenfunctions $\psi_{j}$. The eigenfunctions $\psi_{j}$ form an orthonormal basis in $L^{2}(\mathcal{M})$ [28. We may order eigenvalues as $0=\lambda_{0}<\lambda_{1} \leq \lambda_{2} \cdots$ and corresponding eigenfunctions as $\psi_{0}, \psi_{1}, \psi_{2}, \cdots$.

heat kernel $K_{\sigma}(p, q)$ is then analytically given as

$$
K_{\sigma}(p, q)=\sum_{j=0}^{\infty} e^{-\lambda \sigma} \psi_{j}(p) \psi_{j}(q),
$$

where $\sigma$ is the bandwidth of the kernel [29]. Heat kernel smoothing of $Y$ is given analytically defined as

$$
K_{\sigma} * Y(p)=\sum_{j=0}^{\infty} e^{-\lambda \sigma} \beta_{j} \psi_{j}(p),
$$

where $\beta_{j}=\left\langle Y, \psi_{j}\right\rangle$ are Fourier coefficients. The heat kernel smoothing (4) is taken as an estimate for the unknown signal $\theta$. Since the expansion (4) is a unique solution to isotropic heat diffusion, we can avoid the need to solve the diffusion using less stable numerical schemes such as the finite difference method 2930 .

\subsection{Numerical Implementation}

As the closed form expression for the eigenfunctions of the Laplace-Beltrami operator on an arbitrary curved surface is unknown, the eigenfunctions are numerically calculated by discretizing the Laplace-Beltrami operator. To solve the eigensystem (2), we need to discretize it on a triangular mesh using the Cotan discretization [31,32. Using the Cotan discretization, (2) is linearized as the generalized eigenvalue problem:

$$
\mathbf{C} \psi=\lambda \mathbf{A} \psi
$$




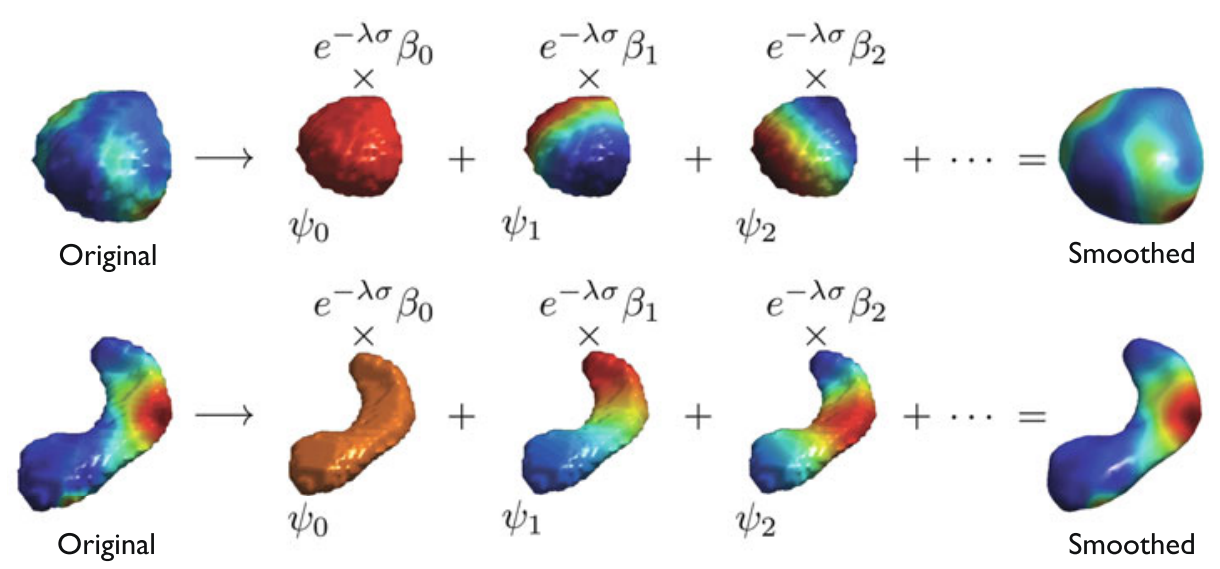

Fig. 3. Illustration of heat kernel smoothing. By summing the Laplace-Beltrami eigenfunctions, we smooth out functional measurements on surfaces. The left most surfaces are the noisy original surfaces with the displacement length. First three eigenfunctions $\psi_{0}, \psi_{1}, \psi_{2}$ are shown in the middle. The right most surfaces are the results of summation with $\sigma=0.5$.

where $\mathbf{C}$ is the stiffness matrix, $\mathbf{A}$ is the mass matrix and $\boldsymbol{\psi}=\left(\psi\left(p_{1}\right), \cdots, \psi\left(p_{n}\right)\right)^{\prime}$ is the unknown eigenfunction evaluated at $n$ mesh vertices. A first few eigenfunctions for the subcortical surfaces are shown in Fig. 3 .

In this study, we have chosen the bandwidth $\sigma=0.5$ and used the finite eigenfunction expansion using up to 1,000 basis (Fig. 3). We smoothed the length of displacement vector field and the coordinates of template surfaces as well.

Once we obtained the basis functions $\psi_{j}$ numerically, we need to estimate the Fourier coefficients $\beta_{j}$. It can be shown that the Fourier coefficients can be estimated as

$$
\beta_{j}=\mathbf{Y}^{\prime} \mathbf{A} \psi_{j}
$$

where $\mathbf{Y}=\left(Y\left(p_{1}\right), \cdots, Y\left(p_{n}\right)\right)^{\prime}$ and $\boldsymbol{\psi}_{j}=\left(\psi_{j}\left(p_{1}\right), \cdots, \psi_{j}\left(p_{n}\right)\right)^{\prime}$ [33].

The MATLAB code for computing the eigenfunctions and performing heat kernel smoothing is available at http://brainimaging.waisman.wisc. edu/ chung/lb/.

\subsection{Validation}

The heat kernel smoothing framework is validated on a unit sphere where the Laplace-Beltrami eigenfunctions are exactly given as spherical harmonics. We used a spherical mesh with 40,962 uniformly sampled mesh vertices. Let $Y_{l m}$ be the spherical harmonic of degree $l$ and order $m$ [34]. Due to the multiplicity, there are $2 l+1$ eigenfunctions $Y_{l,-l}, \cdots, Y_{l, l}$ corresponding to the same eigenvalue $l(l+1)$. Further, any linear combination $\sum_{m=-l}^{l} \beta_{l m} Y_{l m}$ is an eigenfunction as well. So it is not possible to validate the accuracy of the obtained eigenfunctions. Therefore, we only checked if solving (5) produces the expected eigenvalues. 

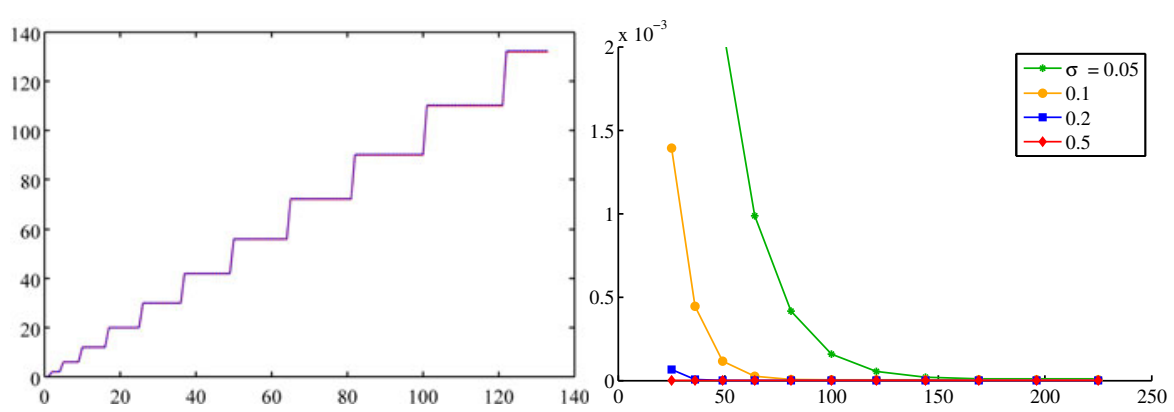

Fig. 4. Left: 133 eigenvalues are numerically computed (blue dotted) and compared against the ground truth (red solid) $\lambda_{l}=l(l+1)$ for up to degree $l=11$. Right: The plot of the root mean squared errors (RMSE) of computed heat kernel over the number of eigenfunctions used (horizontal) for bandwidths $0.05,0.1,0.2$ and 0.5 . As the number of eigenfunctions increases, our implementation converge to the ground truth.

Fig. 4 shows the 133 computed eigenvalues compared against the ground truth. The maximum possible relative error is $0.0032(0.32 \%)$.

We also checked the accuracy of the constructed heat kernel. On a unit sphere, the heat kernel is given by

$$
K_{\sigma}(p, q)=\sum_{l=0}^{\infty} \sum_{m=-l}^{l} e^{-l(l+1) \sigma} Y_{l m}(p) Y_{l m}(q) .
$$

We have taken the degree $l=85$ expansion as the ground truth and compared it to the numerically constructed heat kernel. The RMSE of heat kernel against the ground truth was computed for various bandwidth between 0.05 and 0.5 (Fig. 4). The rate of convergence depends on the bandwidth. As the number of eigenfunctions increases, the constructed heat kernel converge to the ground truth quickly. Beyond 150 eigenfunctions, the reconstruction error is negligible.

\section{Results: General Linear Models on Surface Shapes}

\subsection{Traditional Volumetric Analysis}

In the traditional volumetric approach, the volumes of amygdala and hippocampus binary mask were simply computed by counting the number of voxels within the mask. In order to account for the effect of intersubject variability in brain size, the brain volume excluding cerebellum was computed and covariated in general linear models.

The brain volume is significantly correlated with the amygdala (left: $r=0.55$, $p<10^{-5}$; right: $r=0.49, p<10^{-4}$ ) and the hippocampus volumes (left: $r=0.59$, $p<10^{-7}$; right: $\left.r=0.63, p<10^{-8}\right)$. Since amygdala and hippocampus volumes are dependent on the whole brain volume, we really need to factor out the brain volume in the general liner models. 
We model the Volume of amygdala and hippocampus as

$$
\text { Volume }=\beta_{1}+\beta_{2} \cdot \text { Brain }+\beta_{3} \cdot \text { Age }+\beta_{4} \cdot \text { Gender }+\epsilon
$$

where $\epsilon$ is zero mean Gaussian noise and Brain is the total brain volume. The age and gender effects are determined by testing the significance of parameters $\beta_{3}$ and $\beta_{4}$ at $\alpha=0.01$. The results are displayed in Figure 5 .
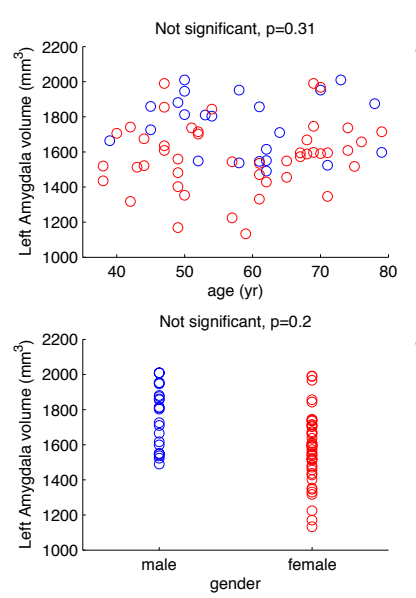
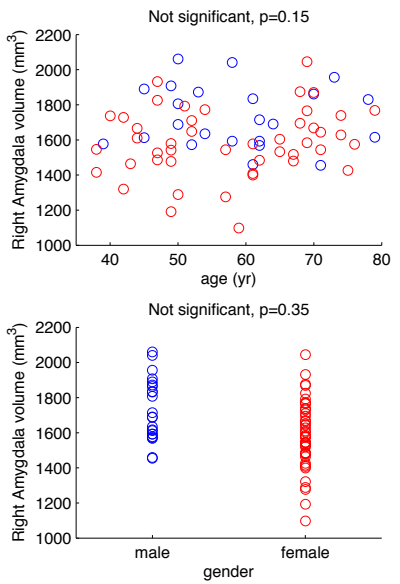

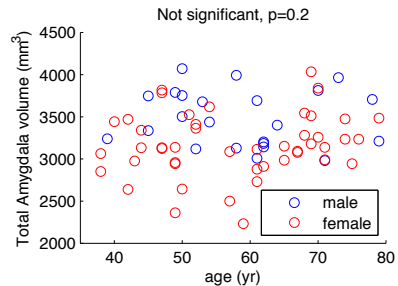

Not significant, $p=0.2$

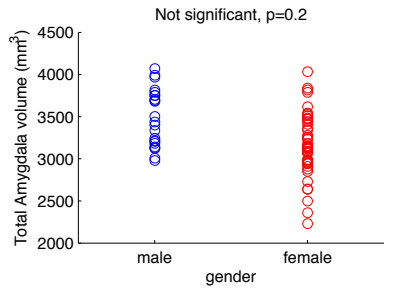

Fig. 5. Scatterplots of left, right and combined amygdala volumes over age (top) and gender (bottom)
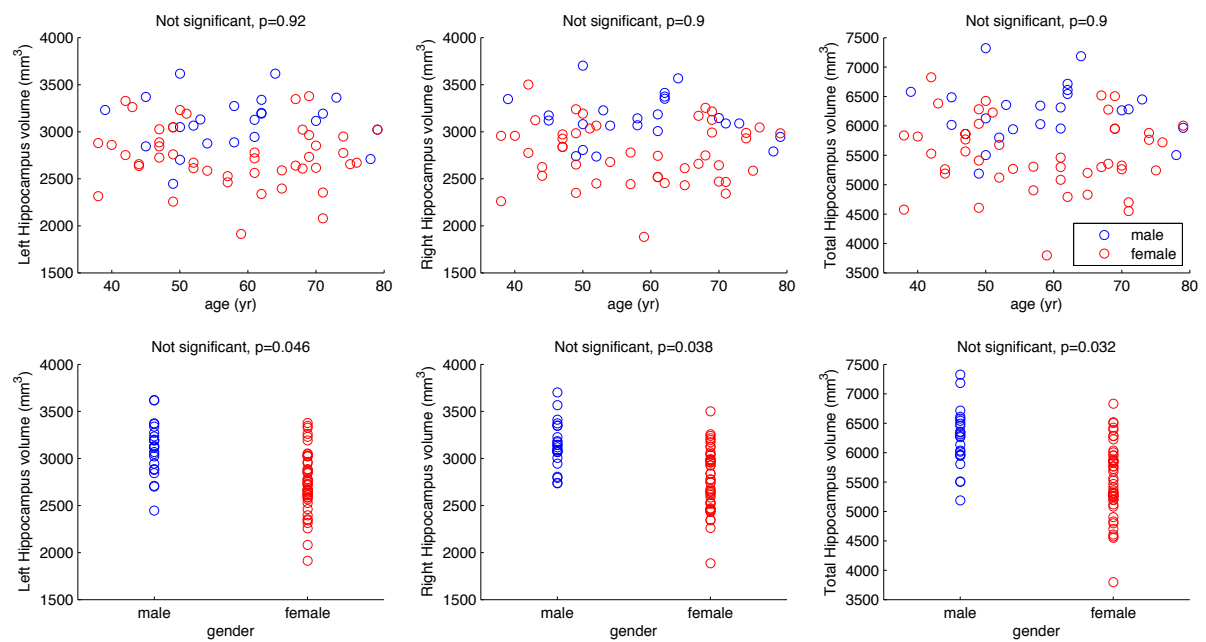

Fig. 6. Scatterplots of the volume of left, right and total hippocampus over age (top) and gender (bottom) 
For the amygdala volume, we did not find a significant effect of age (left $p=$ 0.31 ; right $p=0.15$; combined $p=0.20$ ) nor gender (left $p=0.20$; right $p=0.35$; combined $p=0.25)$ For the hippocampus volume, we did not find a significant effect of age (left $p=0.92$; right $p=0.90$; total $p=0.90$ ) nor gender (left $p=0.05$; right $p=0.04$; total $p=0.03)$.

Since our results are based on the volume of the whole amygdala and hippocampus, it is still unclear if there are any localized shape differences within the parts of amygdala and hippocampus.

\subsection{Localized Subcortical Shape Analysis}

The length of displacement vector fields along the template surfaces were computed and smoothed as described in section 2. Then Length is regressed over the total brain volume and other variables:

$$
\text { Length }=\beta_{1}+\beta_{2} \cdot \text { Brain }+\beta_{3} \cdot \text { Age }+\beta_{4} \cdot \text { Gender }+\epsilon
$$

where $\epsilon$ is zero mean Gaussian noise. The age and gender effects are determined by testing the significance of parameters $\beta_{3}$ and $\beta_{4}$ at $\alpha=0.01$. We used SurfStat MATLAB toolbox (http://galton.uchicago.edu/faculty/InMemoriam/ worsley/research/surfstat/), for the statistical analysis and multiple comparison correction. The details on the SurfStat package is given in [34]. The results are displayed in Figure 7.

a) age effect

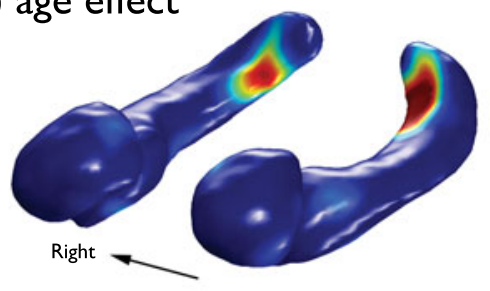

b) gender effect

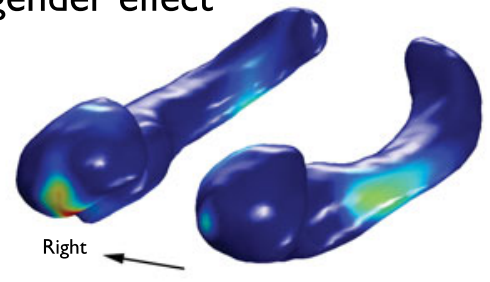

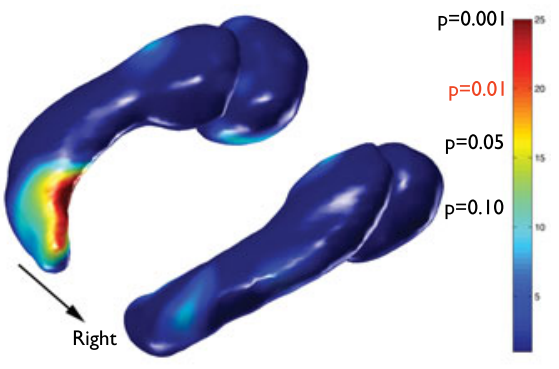

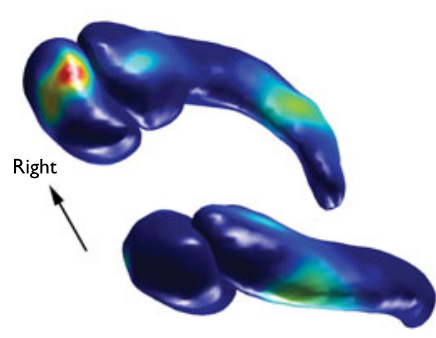

Fig. 7. F-statistic maps on the amygdala and hippocampus surfaces showing the age (a) and gender (b) effects with corresponding $p$-values indicated. The posterior regions of the both left and right hippocampi show a significant age effect. The ventral region of the right amygdala shows a significant gender effect. 
Age effect. We found the region of significant effect of age on the posterior part of hippocampi (left: $\max \mathrm{F}=39.43, p<10^{-5}$; right: $\max \mathrm{F}=23.11, p=0.002$ ) Particularly, on the caudal regions of the left and right hippocampi, we found highly localized signals. It is consistent with other shape modeling studies on hippocampus 35136. We did not find any age effects on the amygdala surface at $\alpha=0.01$.

Gender effect. We found a highly focalized region of gender effect on the inferior part of the right amygdala ( $\max \mathrm{F}=24.66, p<0.001$ ). In particular, the gender effect is focused around the ventral part of laterobasal group [37.

We did not find any significant gender effects on the left amygdala and hippocampi.

\section{Conclusion}

We have presented a new subcortical structure shape modeling framework using heat kernel smoothing constructed with the Laplace-Beltrami eigenfunctions. The proposed framework demonstrated higher sensitivity in modeling shape variations compared to the traditional volumetric analysis. The ability to localize subtle morphological difference may provide an anatomical evidence for the functional organization within human subcortical structures.

\section{References}

1. LeDoux, J.: The amygdala. Current Biology 17(20), R868-R874 (2007)

2. Alvarez, P., Squire, L.R.: Memory consolidation and the medial temporal lobe: a simple network model. Proceedings of the National Academy of Sciences 91(15), 7041-7045 (1994)

3. Miller, A., Alston, R., Corsellis, J.: Variation with age in the volumes of grey and white matter in the cerebral hemispheres of man: measurements with an image analyser. Neuropathology and Applied Neurobiology 6(2), 119-132 (1980)

4. Good, C., Johnsrude, I., Ashburner, J., Henson, R., Friston, K., Frackowiak, R.: A voxel-based morphometric study of ageing in 465 normal adult human brains. NeuroImage 14(1), 21-36 (2001)

5. Walhovd, K., Westlye, L., Amlien, I., Espeseth, T., Reinvang, I., Raz, N., Agartz, I., Salat, D., Greve, D., Fischl, B., et al.: Consistent neuroanatomical age-related volume differences across multiple samples. Neurobiology of Aging (2009)

6. Bigler, E., Blatter, D., Anderson, C., Johnson, S., Gale, S., Hopkins, R., Burnett, B.: Hippocampal volume in normal aging and traumatic brain injury. American Journal of Neuroradiology 18(1), 11 (1997)

7. Du, A., Schuff, N., Chao, L., Kornak, J., Jagust, W., Kramer, J., Reed, B., Miller, B., Norman, D., Chui, H., et al.: Age effects on atrophy rates of entorhinal cortex and hippocampus. Neurobiology of Aging 27(5), 733-740 (2006)

8. Walhovd, K., Fjell, A., Reinvang, I., Lundervold, A., Dale, A., Eilertsen, D., Quinn, B., Salat, D., Makris, N., Fischl, B.: Effects of age on volumes of cortex, white matter and subcortical structures. Neurobiology of Aging 26(9), 1261-1270 (2005) 
9. Sullivan, E., Marsh, L., Mathalon, D., Lim, K., Pfefferbaum, A.: Age-related decline in mri volumes of temporal lobe gray matter but not hippocampus. Neurobiology of Aging 16(4), 591-606 (1995)

10. Sullivan, E., Marsh, L., Pfefferbaum, A.: Preservation of hippocampal volume throughout adulthood in healthy men and women. Neurobiology of Aging 26(7), 1093 (2005)

11. Good, C., Johnsrude, I., Ashburner, J., Henson, R., Friston, K., Frackowiak, R.: Cerebral asymmetry and the effects of sex and handedness on brain structure: a voxel-based morphometric analysis of 465 normal adult human brains. NeuroImage $14(3), 685-700(2001)$

12. Gur, R.C., Gunning-Dixon, F., Bilker, W.B., Gur, R.E.: Sex differences in temporolimbic and frontal brain volumes of healthy adults. Cerebral Cortex 12(9), 998-1003 (2002)

13. Chung, M.K., Worsley, K.J., Paus, T., Cherif, D.L., Collins, C., Giedd, J., Rapoport, J.L., Evans, A.C.: A unified statistical approach to deformation-based morphometry. NeuroImage 14, 595-606 (2001)

14. Adler, R.: On excursion sets, tube formulas and maxima of random fields. Annals of Applied Probability, 1-74 (2000)

15. Worsley, K., Marrett, S., Neelin, P., Vandal, A., Friston, K., Evans, A., et al.: A unified statistical approach for determining significant signals in images of cerebral activation. Human Brain Mapping 4(1), 58-73 (1996)

16. Worsley, K., Evans, A., Marrett, S., Neelin, P.: A three-dimensional statistical analysis for $\mathrm{CBF}$ activation studies in human brain. Journal of Cerebral Blood Flow and Metabolism 12, 900 (1992)

17. Smith, S.: Fast robust automated brain extraction. Human Brain Mapping 17(3), 143-155 (2002)

18. Avants, B., Epstein, C., Grossman, M., Gee, J.: Symmetric diffeomorphic image registration with cross-correlation: Evaluating automated labeling of elderly and neurodegenerative brain. Medical Image Analysis 12(1), 26-41 (2008)

19. Lorensen, W., Cline, H.: Marching cubes: A high resolution 3D surface construction algorithm. ACM Siggraph Computer Graphics 21(4), 163-169 (1987)

20. Chung, M.K., Worsley, K.J., Robbins, S., Paus, T., Taylor, J., Giedd, J.N., Rapoport, J.L., Evans, A.C.: Deformation-based surface morphometry applied to gray matter deformation. NeuroImage 18, 198-213 (2003)

21. Perona, P., Malik, J.: Scale-space and edge detection using anisotropic diffusion. IEEE Transactions on Pattern Analysis and Machine Intelligence 12(7), 629-639 (1990)

22. Andrade, A., Kherif, F., Mangin, J., Worsley, K., Paradis, A., Simon, O., Dehaene, S., Le Bihan, D., Poline, J.B.: Detection of fmri activation using cortical surface mapping. Human Brain Mapping 12, 79-93 (2001)

23. Sochen, N., Kimmel, R., Malladi, R.: A general framework for low level vision. IEEE Transactions on Image Processing 7(3), 310-318 (1998)

24. Malladi, R., Ravve, I.: Fast Difference Schemes for Edge Enhancing Beltrami Flow. In: Heyden, A., Sparr, G., Nielsen, M., Johansen, P. (eds.) ECCV 2002. LNCS, vol. 2350, pp. 343-357. Springer, Heidelberg (2002)

25. Tang, B., Sapiro, G., Caselles, V.: Direction diffusion. In: The Proceedings of the Seventh IEEE International Conference on Computer Vision, vol. 2, pp. 1245-1252 (1999)

26. Taubin, G.: Geometric signal processing on polygonal meshes. In: Eurographics 2000 - State of the Art Report (2000) 
27. Joshi, A., Shattuck, D., Thompson, P., Leahy, R.: A parameterization-based numerical method for isotropic and anisotropic diffusion smoothing on non-flat surfaces. IEEE Transactions on Image Processing 18(6), 1358-1365 (2009)

28. Lévy, B.: Laplace-beltrami eigenfunctions towards an algorithm that understands geometry. In: IEEE International Conference on Shape Modeling and Applications, SMI 2006, p. 13. IEEE (2006)

29. Chung, M., Robbins, S., Dalton, K., Davidson, R., Alexander, A., Evans, A.: Cortical thickness analysis in autism with heat kernel smoothing. NeuroImage 25(4), 1256-1265 (2005)

30. Tasdizen, T., Whitaker, R., Burchard, P., Osher, S.: Geometric surface smoothing via anisotropic diffusion of normals. In: IEEE Visualization Conference (2002)

31. Chung, M., Taylor, J.: Diffusion smoothing on brain surface via finite element method. In: IEEE International Symposium on Biomedical Imaging: Nano to Macro, pp. 432-435. IEEE (2004)

32. Qiu, A., Bitouk, D., Miller, M.: Smooth functional and structural maps on the neocortex via orthonormal bases of the laplace-beltrami operator. IEEE Transactions on Medical Imaging 25(10), 1296-1306 (2006)

33. Zhang, H., van Kaick, O., Dyer, R.: Spectral methods for mesh processing and analysis. In: Eurographics 2007-State of the Art Reports, pp. 1-22 (2007)

34. Chung, M.K., Worsley, K.J., Nacewicz, B.M., Dalton, K.M., Davidson, R.J.: General multivariate linear modeling of surface shapes using surfstat. NeuroImage 53(2), 491-505 (2010)

35. Qiu, A., Miller, M.: Multi-structure network shape analysis via normal surface momentum maps. NeuroImage 42(4), 1430-1438 (2008)

36. Xu, Y., Valentino, D., Scher, A., Dinov, I., White, L., Thompson, P., Launer, L., Toga, A.: Age effects on hippocampal structural changes in old men: the haas. NeuroImage 40(3), 1003-1015 (2008)

37. Amunts, K., Kedo, O., Kindler, M., Pieperhoff, P., Mohlberg, H., Shah, N., Habel, U., Schneider, F., Zilles, K.: Cytoarchitectonic mapping of the human amygdala, hippocampal region and entorhinal cortex: intersubject variability and probability maps. Anatomy and Embryology 210(5), 343-352 (2005) 